MODELING, IDENTIFICATION AND CONTROL, 1988, VOL. 9, NO. 1, 3-15

doi:10.4173/mic.1988.1.1

\title{
On-line component estimation and analyser calibration for a complex industrial processing plant using Kalman filtering
}

\author{
OLA NORDERHAUG, $\uparrow$ JENS G. BALCHEN, $\uparrow$ TOR ONSHUS $\uparrow$
}

Keywords: Kalman filtering, estimation, calibration, chemical analysis

\begin{abstract}
The paper presents an on-line analyser system based on a Kalman filtering technique. The system is implemented on a computer, and tested against an analyser simulator. A mathematical model for the analyser is also determined since an extended Kalman filter can perform both component estimation and analyser calibration, accuracy in the analysis is improved.
\end{abstract}

\section{Introduction}

On-line multi-component analysis of chemical composition has considerable economic potential in the chemical industry. At Norsk Hydro's plant near Porsgrunn, Norway, an automatic on-line analyser is being used to determine the concentration of a number of different components in a complex product. By modeling the physical phenomena involved in the different processes in the analyser, a Kalman filter can provide an optimal estimation of the chemical components and can also be used for the calibration of the analyser.

In this paper a Kalman filter (1) for two of the sensors in the analyser is designed. The method will be examined by computer simulation experiments.

\section{System description}

\subsection{General}

With a fixed frequency, samples are removed from the process stream for analysis using a liquid analyser.

The analyser employs standard solutions of known composition. Both the samples and the standard solutions are treated identically in the analyser. The liquid is transported to the sensors by air-segmented continuous flow in a sequence such as that shown in Fig. 1.

The sensors used are ion-selective electrodes which have a Nernstian response described by the formula

$$
E=E_{0}+2 \cdot 3 \frac{R T}{z_{i} F} \log \left(a_{i}+\sum_{j=1}^{n} k_{i j} a_{j}^{z_{j} / z_{j}}\right)
$$

Received June 11987

$\dagger$ Division of Engineering Cybernetics, The Norwegian Institute of Technology, 7034 Trondheim-NTH, Norway.

This paper was presented at the 1986 American Control Conference. 


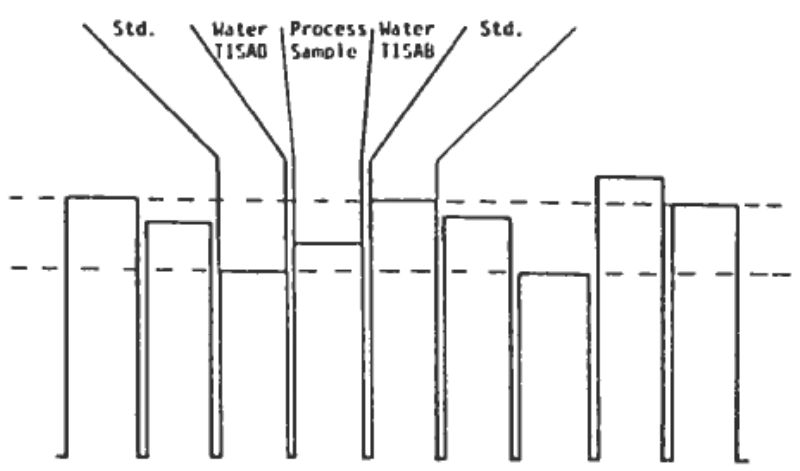

Figure 1. The flow sequence.

where

$E$ is the measured potential

$E_{0}$ is a temperature-dependent constant

$2 \cdot 3 \frac{R T}{z_{i} F}$ is the Nernst factor

$a_{i}$ is the activity of the ion being measured

$a_{j}$ is the activity of the $j$ th interfering ion

$z_{i}, z_{j}$ are the charges of the ion being measured and the $j$ th interfering ion respectively

$k_{i j}$ is the selectively constant for the $j$ th ion

Ion-selective electrodes measure ion activities and not ion concentrations. However activity is equal to the concentration multiplied by the activity coefficient, or $a_{i}=$ $c_{i} f_{i}$. The activity coefficient, $f_{i}$, is a function of the ionic strength of the solution. In our system a Total Ionic Strength Adjustment Buffer, TISAB, is added. This raises the ionic strength to a level where the activity coefficients of the ions in the sample will be identical with those in the standards. The activity coefficient can now be regarded as a constant. Thus a single calibration curve will suffice to determine the concentration of a particular ion.

The electrodes have a drift which, apart from the change in temperature, is due to the change of the reference potentials as well as the electrode responses. The

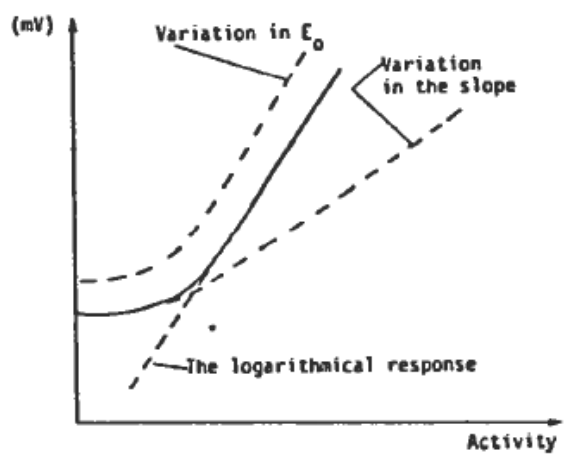

Figure 2. The static response of an ion-selective electrode. 
sources of the drift consist of: the inner reference electrodes, the membrane and its asymmetrical potential, the junction potential and other reference electrodes.

A typical response curve for an ion-selective electrode is indicated in Fig. 2. Drift in the various parameters is indicated by dashed lines.

In this paper we will only deal with two of the +ion-selective electrodes in the analyser, the A-electrode and the B-electrode. We assume that the A-ion is the only interfering ion in the B-electrode, and that the B-ion is the only interfering ion in the A-electrode. However there are many other species present, but they do not affect the measurements significantly.

\subsection{Mathematical modeling}

In the actual system there is a continuous flow of pulses with different compositions. The sequence in Fig. 1 does not give the sensors enough time to reach steady state signals. Similarly the signal from the preceeding pulse is not entirely swamped out when the pulse arrives for analysis. This carryover from one pulse to the next is due to three phenomena: the diffusion of ion in the membrane takes time, the washout in the flow cell is imperfect, and a thin film from the preceding pulse is mixed with the next pulse in the transport system.

These phenomena can be modeled as time constants, resulting in a simple mathematical model for the dynamics of the sensor:

$$
\left.\begin{array}{l}
\dot{x}_{1}(t)=\frac{1}{T_{i 1}}\left[k_{01}+k_{1} \log \left(c_{1}(t)+k_{12} c_{2}(t)+c_{0}\right)-x_{1}(t)\right] \\
\dot{x}_{2}(t)=\frac{1}{T_{i 2}}\left[k_{02}+k_{2} \log \left(c_{2}(t)+k_{21} c_{1}(t)+c_{0}\right)-x_{2}(t)\right]
\end{array}\right\}
$$

where

$x_{1}(t)$ the measured potential of the A-electrode

$x_{2}(t)$ the measured potential of the B-electrode

$c_{1}(t)$ the concentration of A-ions

$c_{2}(t)$ the concentration of B-ions

$k_{01}, k_{02}$ constants corresponding to $E_{0}$ in (1)

$k_{1}, k_{2}$ constants corresponding to the Nernst factor

$k_{12}, k_{21}$ selectivity constants

$T_{i 1}, T_{i 2}$ are time constants for the rising $(i=1)$ and the falling edge $(i=2)$ for the A-electrode and the B-electrode respectively.

$c_{0}=0.510^{-5}$ is a 'constant contamination' used in the simulations to prevent the log-term from converging to $-\infty$.

The following measurement model is used

$$
\left.\begin{array}{l}
y_{1}(t)=x_{1}(t) \\
y_{2}(t)=x_{2}(t)
\end{array}\right\}
$$

Discrete versions of (2) and (3) are employed when implementing the models in a computer system. 


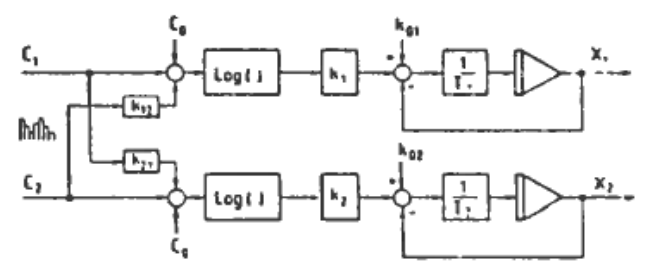

Figure 3. The analyser simulator.

\section{Kalman filter simulations}

\subsection{Simulator}

The Kalman filter is designed and implemented on a digital NORD-10 computer. For purposes of testing and evolution of the Kalman filter, a simulator for generation of realistic analyser-signals has been developed.

The simulator simply uses the model described in (2) and (3) with concentrations $c_{1}(t)$ and $c_{2}(t)$, varying as square-pulses with different amplitudes according to the sequence in Fig. 1. The simulator is shown in Fig. 3.

The time constants were investigated by recording the step response of the analyser. Figure 4, shows the step responses for the A- and B-electrodes. The dashed lines show how the model responds using the time constants indicated in the figures.

The actual time constants fluctuated along the curves, which can be modeled as illustrated in Fig. 4. However in the simulations we opted for fixed time constants. An extension to include varying time constants is possible as long as these variations can be modeled.

Figure 5 shows the measurements generated by the simulator for one of the electrodes. In this simulation the concentrations of the sample pulses were held constant. It can clearly be seen that the sample measurements are influenced by the preceeding sample.

\subsection{Estimation of concentration and calibration of analyser using Kalman-filter}

We are primarily interested in determining the concentrations of the different species in the sample. Due to drift in the ion-selective electrodes it is also necessary to calibrate the analyser. Using a Kalman-filter we can determine the concentrations using the non-linear model described in (2), and calibrate the analyser on-line. The calibration is performed when analysing the standard solutions, while the concentrations are estimated when analysing the process stream components. This is illustrated in Fig. 6.

Using model (2), drift in the analyser is represented by drift in the parameters of the model. The parameters most likely to be drifting are $k_{01}, k_{02}, k_{1}$ and $k_{2}$. The system is therefore augmented with the following state variables:

$$
\begin{array}{ccc}
x_{3}(t)=c_{1}(t), & x_{4}(t)=c_{2}(t), \quad x_{5}(t)=k_{01} \\
x_{6}(t)=k_{02}, & x_{7}(t)=k_{1}, \quad x_{8}(t)=k_{2}
\end{array}
$$

These augmented variables are modeled by the simple differential equations:

$$
\dot{x}_{i}=0, \quad i=3,4,5,6,7,8
$$



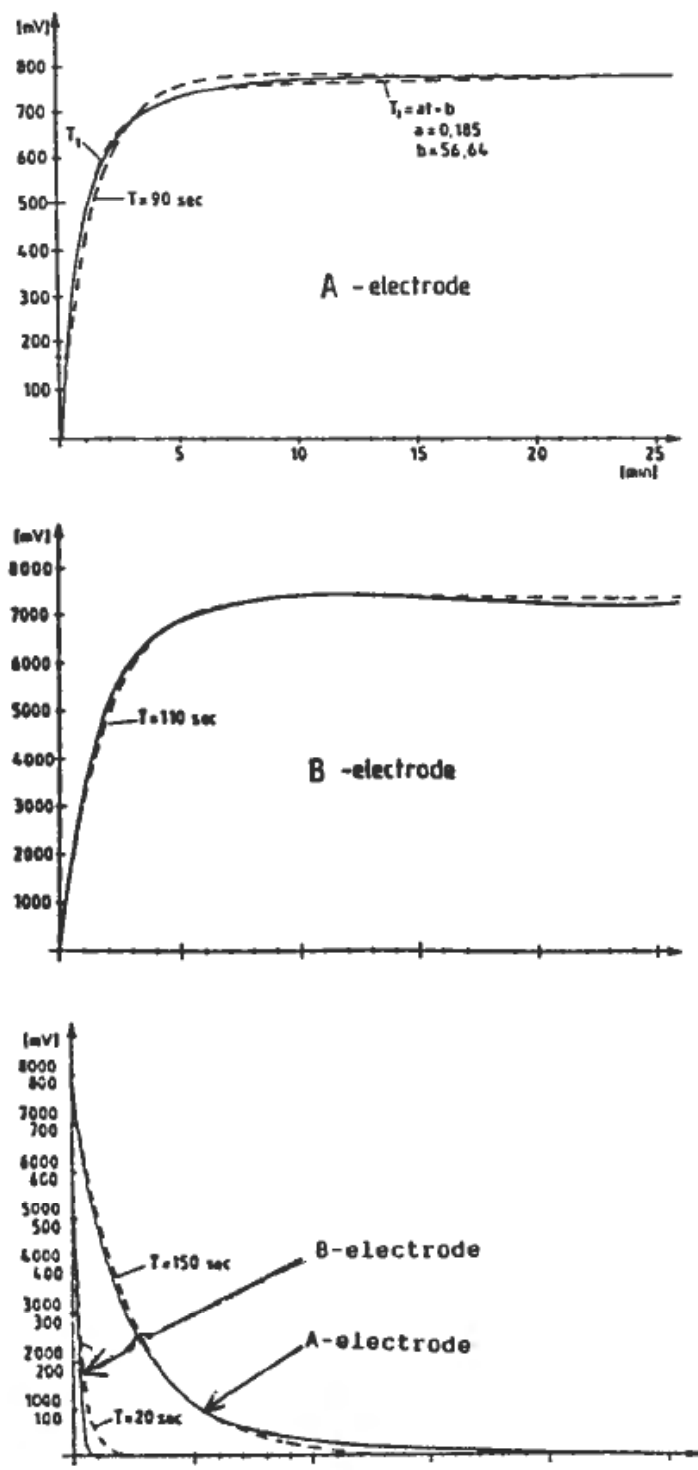

Figure 4. The step response of the analyser.

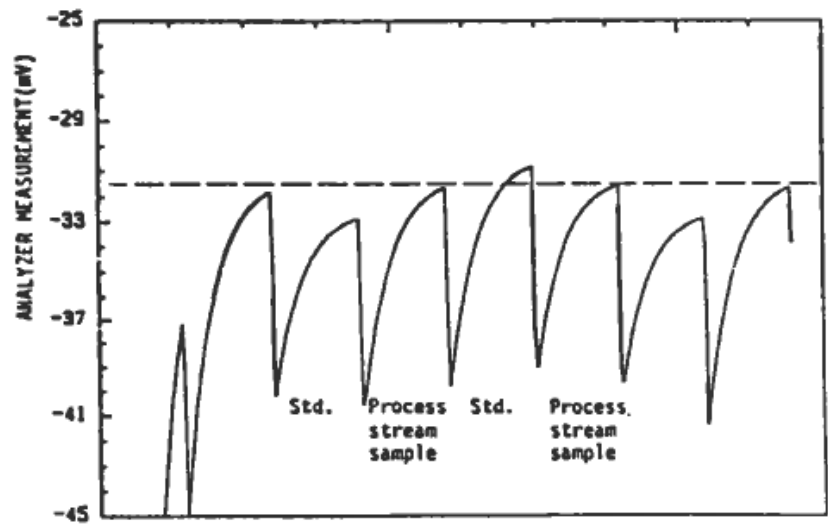

Figure 5. Measurements generated by the simulator. 


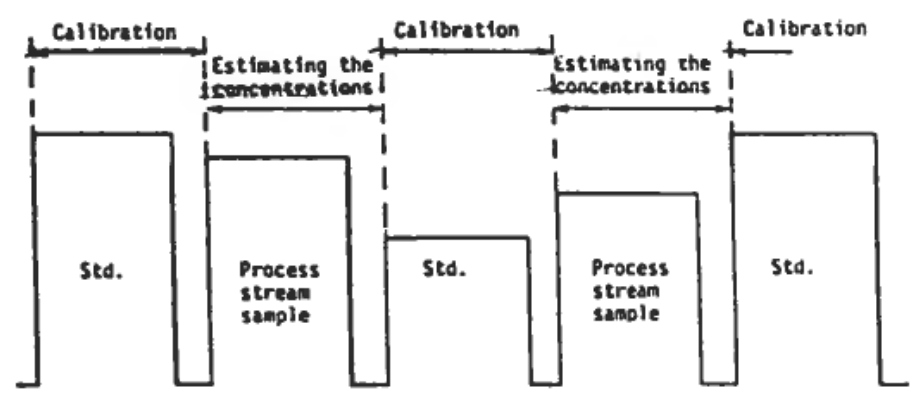

Figure 6. Component estimation and analyser calibration.

The total augmented model thus becomes:

$$
\left.\begin{array}{l}
\dot{x}_{1}=\frac{1}{T_{i 1}}\left[x_{5}+x_{7} \log \left(x_{3}+k_{12} x_{4}+c_{0}\right)-x_{1}\right] \\
\dot{x}_{2}=\frac{1}{T_{i 2}}\left[x_{6}+x_{8} \log \left(x_{4}+k_{21} x_{3}+c_{0}\right)-x_{2}\right] \\
\dot{x}_{3}=0, \quad \dot{x}_{4}=0, \quad \dot{x}_{5}=0, \quad \dot{x}_{6}=0, \quad \dot{x}_{7}=0 \\
\dot{x}_{8}=0, \\
y_{1}=x_{1}, \quad y_{2}=x_{2},
\end{array}\right\}
$$

Based on the model given by Eqns. (5) and (6) an extended Kalman-filter is implemented. The filter equations are given in Appendix I.

When in the calibration modus, the composition of the standard solutions are known and no estimation of the concentrations $\left(x_{3}, x_{4}\right)$ is performed. While estimating the concentrations the parameter-estimates are held constant.

The structure of the Kalman filter is shown in Fig. 7. The switching between calibrating the analyser and estimating the concentrations is illustrated by switches.

Figures 8 to 11 show the performance of the filter when using the following parameters in the simulator and estimator:

$$
\begin{aligned}
\text { Simulator: } \\
k_{01}=100[\mathrm{mV}] \\
k_{02}=100[\mathrm{mV}] \\
k_{1}=56[\mathrm{mV}] \\
k_{2}=56[\mathrm{mV}] \\
T_{11}=90[\mathrm{sec}] \\
T_{21}=150[\mathrm{sec}] \\
T_{12}=110[\mathrm{sec}] \\
T_{22}=20[\mathrm{sec}] \\
k_{12}=0.2 \\
k_{21}=0.01
\end{aligned}
$$

\section{Estimator:}

$$
\begin{aligned}
& T_{11}=90[\mathrm{sec}] \\
& T_{21}=150[\mathrm{sec}] \\
& T_{12}=110[\mathrm{sec}] \\
& T_{22}=20[\mathrm{sec}] \\
& k_{12}=0 \cdot 2 \\
& k_{21}=0 \cdot 01
\end{aligned}
$$

Only parameter estimates for one of the electrodes are shown. The parameter estimates for the other electrodes are similar to those in Fig. 11. 


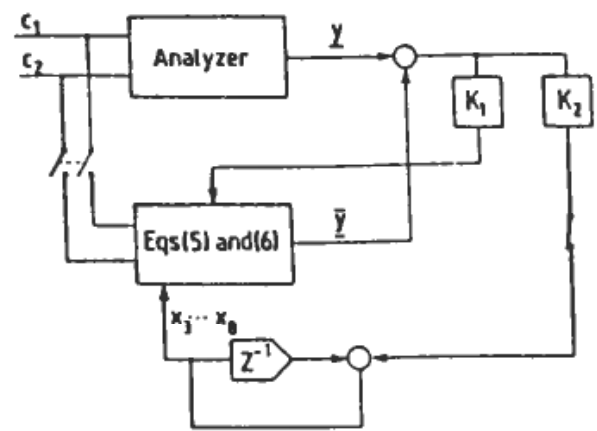

Figure 7. Kalman filter structure.

The accuracy of the concentration estimates depends on the accuracy of the parameter estimates. The selectivity constants $k_{12}$ and $k_{21}$ were assumed to be known. We investigated the filter's sensitivity to wrong assumptions in these constants.

$$
\begin{array}{lll}
\text { Simulator: } k_{12}=0.15, & k_{21}=0.01 \\
\text { Estimator: } k_{12}=0.20, & k_{21}=0.01
\end{array}
$$

Figure 12 shows the results of the simulation. The accuracy of the concentration estimates now depends on how much the unknown concentration deviates from the standard concentrations.

Finally we also tried to estimate the time constants. The model was further augmented by the following variables:

$$
\begin{aligned}
x_{9} & =T_{11} & & (=90) \\
x_{10} & =T_{12} & & (=110) \\
x_{11} & =T_{21} & & (=150) \\
x_{12} & =T_{22} & & (=20)
\end{aligned}
$$

These variables were modeled as before

$$
\dot{x}_{i}=0 \quad i=9,10,11,12
$$

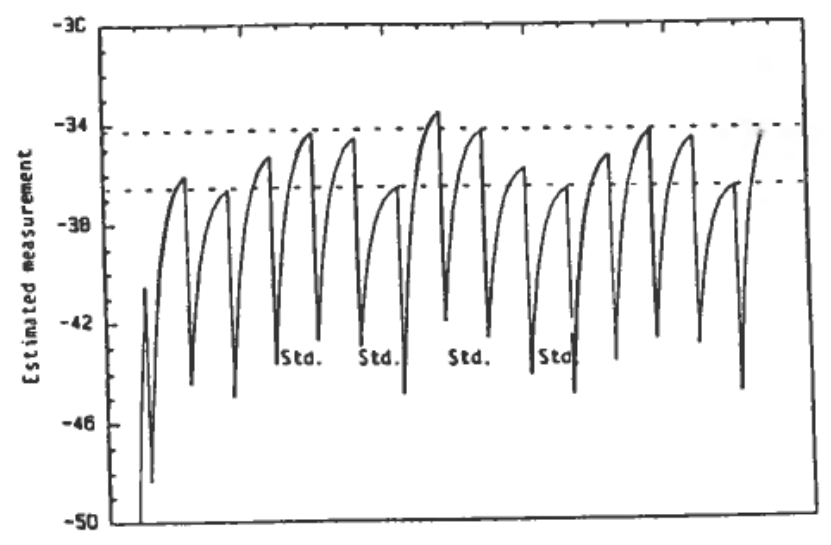

Figure 8. Estimate of $x_{1}, \hat{x}_{1}$. 


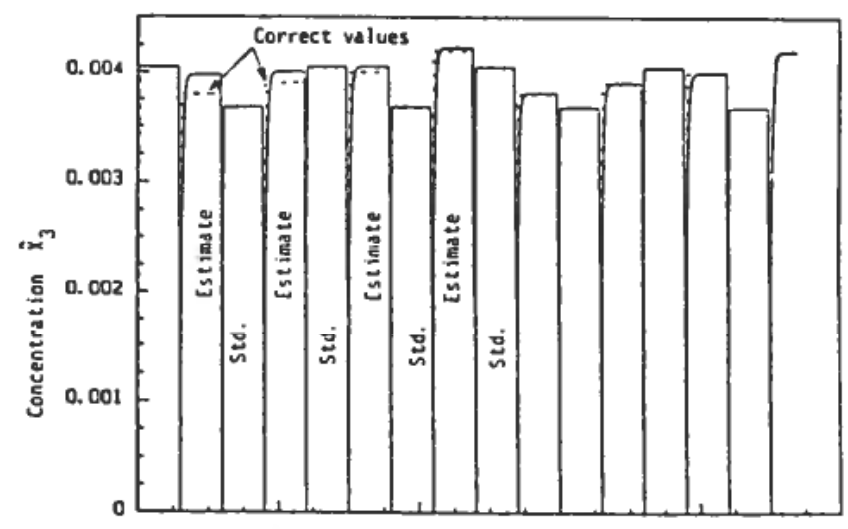

Figure $9(a)$. Estimate concentration, $\hat{x}_{3}$.

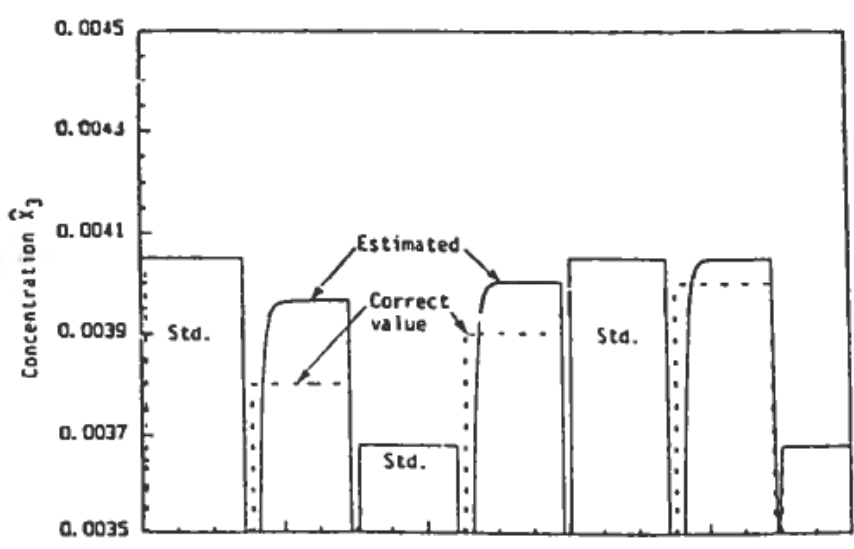

Figure $9(b)$. Estimate concentration, $\hat{x}_{3}$.

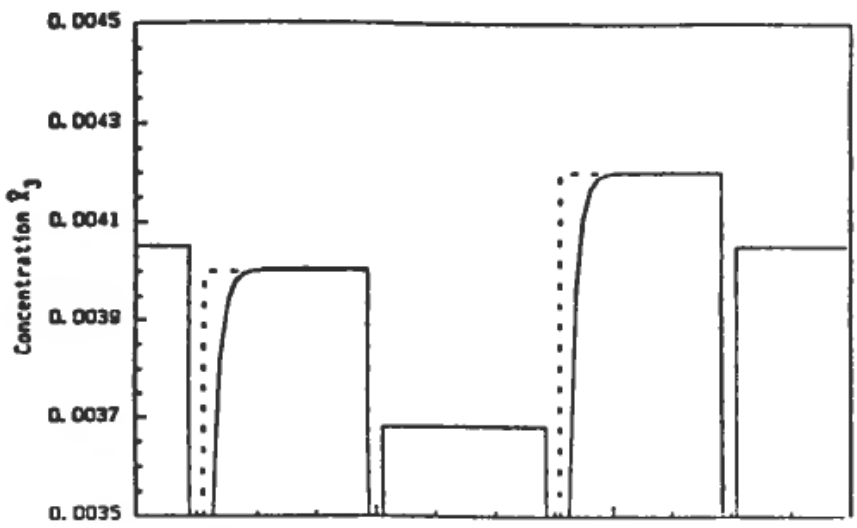

Figure $9(c)$. Estimated concentration, $\hat{x}_{3}$. 

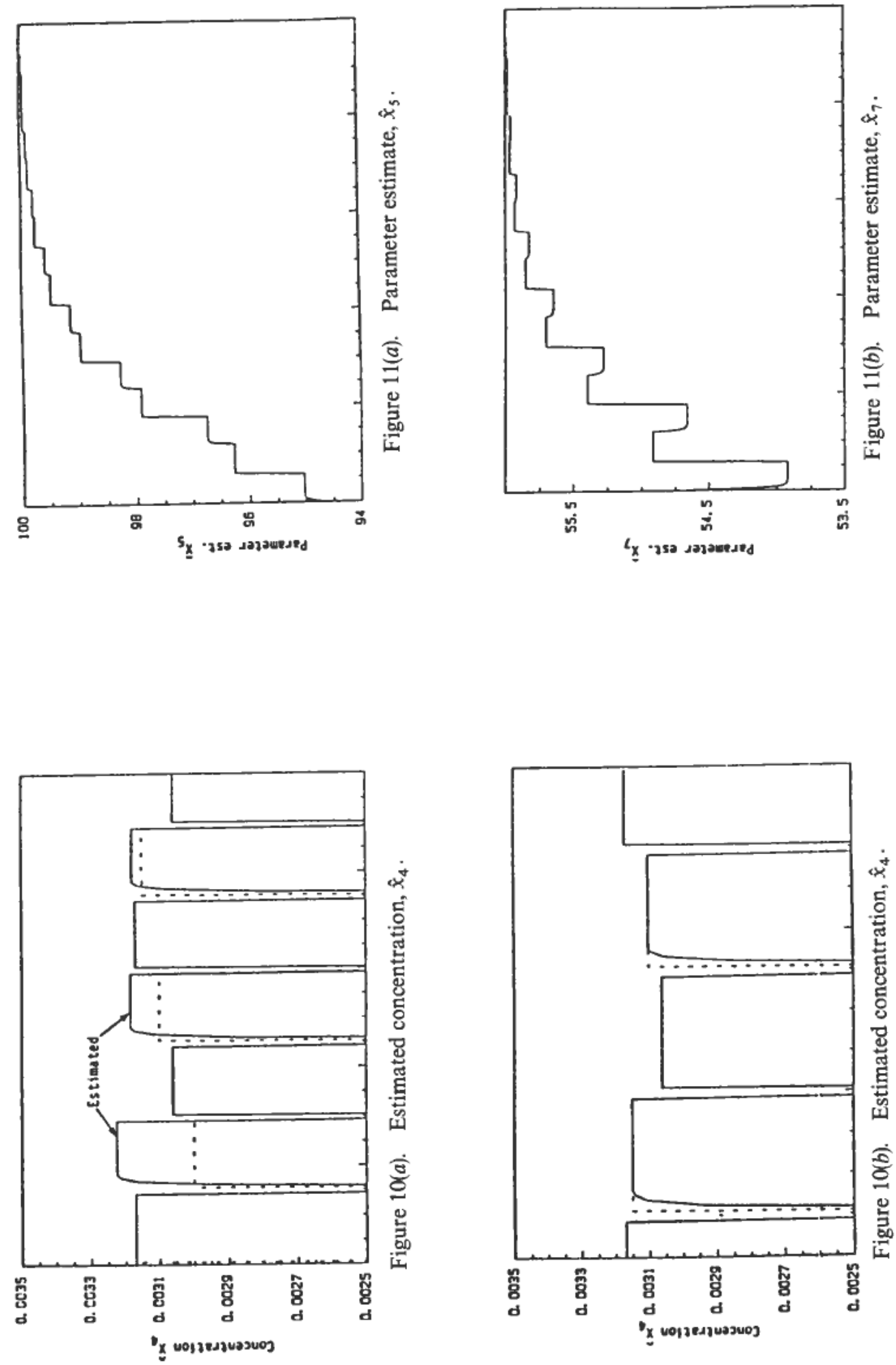


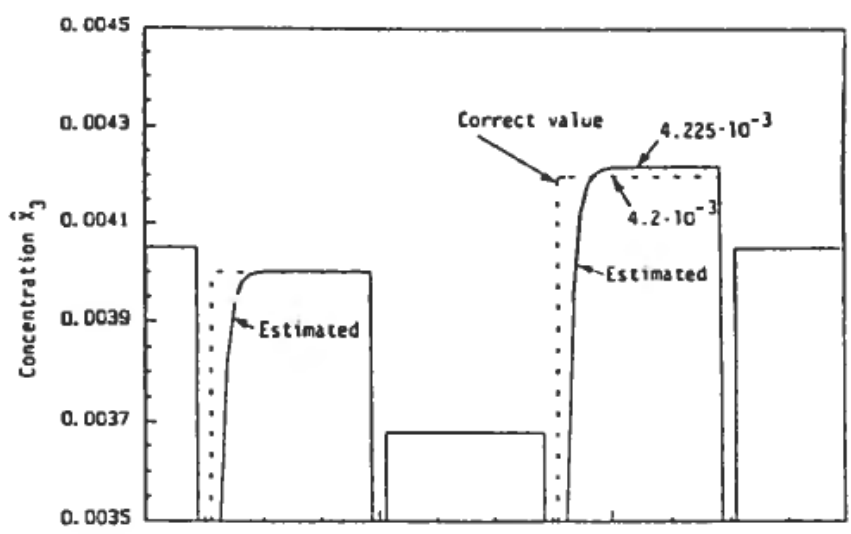

Figure 12(a).

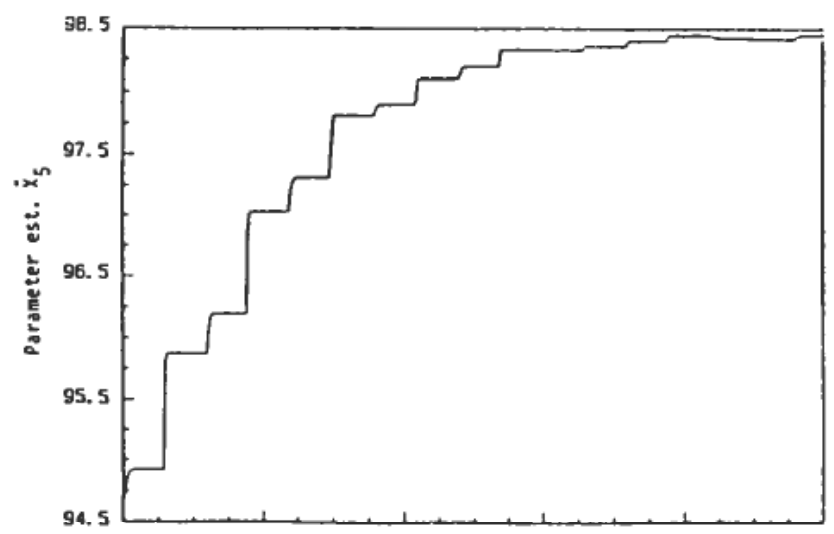

Figure 12(b).

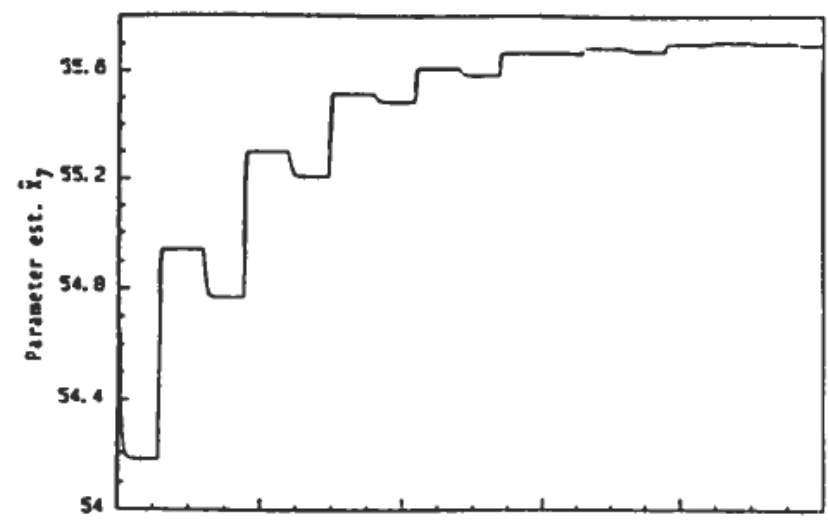

Figure 12(c)

Figure $12(a)-12(f)$. Simulation results with different selectivity constants in the simulator and the estimator ('wrong assumptions in these constants '). 


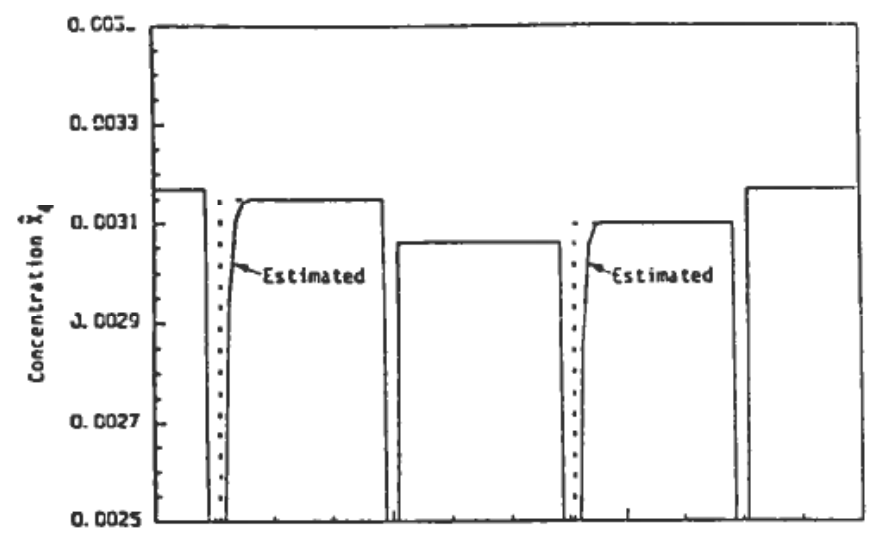

Figure 12(d).

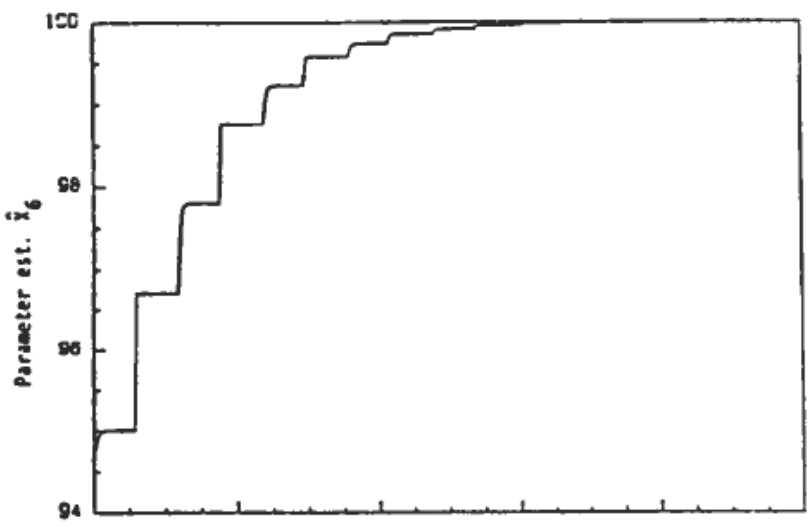

Figure 12(e).

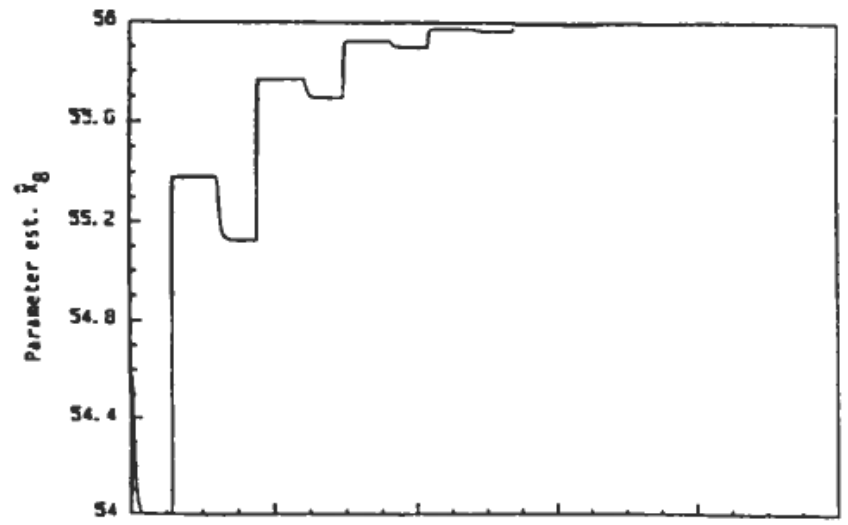

Figure 12(f). 


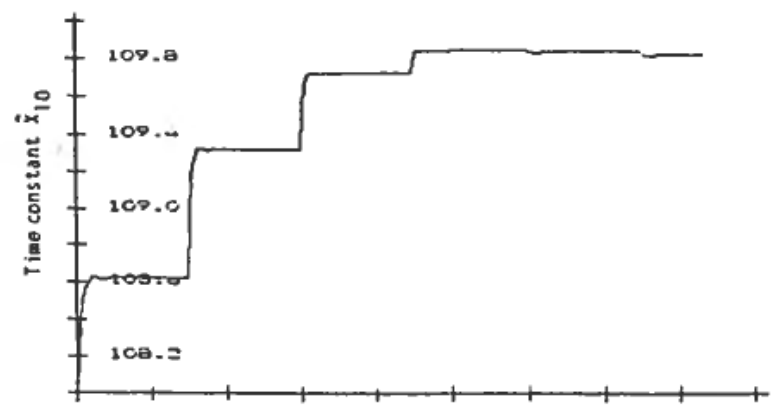

Figure 13(a).

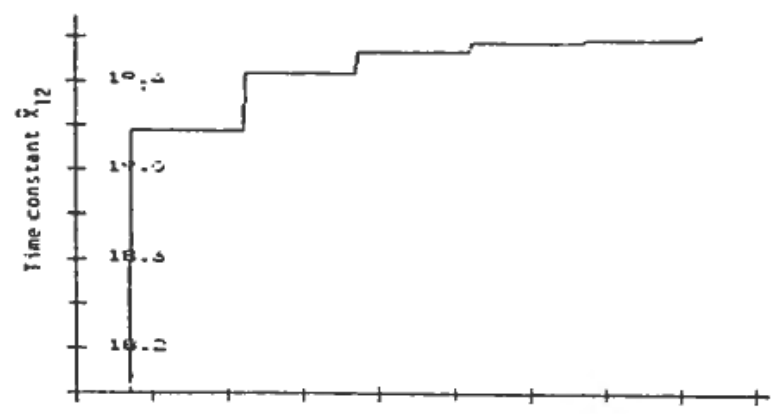

Figure 13(a)-13(b). Estimates of the time constants.

For one of the sensors (B-electrode) the estimates converge to the correct values (Fig. 13). For the other sensor (A-electrode) the estimates diverge. The reason for this could be a programming error. However, it is also possible that the signals from this sensor do not vary enough or that the system is insufficiently excited. This will be investigated further.

\section{Conclusion}

The simulations show that on-line component estimation and analyser calibration in analytical chemistry using Kalman-filtering has a considerable potential. The system gives correct concentration estimates even though the unknown concentrations may differ significantly from the standard concentrations. Further investigations regarding parameter identifiability are necessary.

\section{ACKNOWLEDGMENT}

The authors express their appreciation to Norsk Hydro A/S for permission to publish this material.

\section{REFERENCE}

JAZWINSKI, A. H. (1970). Stochastic processes and filtering theory Academic Press, New York). 


\section{Appendix}

Kalman-filter algorithm

(1) An a priori estimate of the state vector $\overline{\boldsymbol{x}}_{\boldsymbol{k}^{\prime}}$, and its covariance $\bar{X}_{k}$ is assumed to be known.

(2) The filter gain matrix is computed by

$$
K_{k}=\bar{X}_{k} D^{\mathrm{T}}\left(D \bar{X}_{k} D^{\mathrm{T}}+W\right)^{-1}
$$

where

$W$ covariance matrix of the measurement noise

$D$ measurement matrix

$k$ time parameter

(3) The a posteriori state estimate, $\hat{\boldsymbol{x}}_{\boldsymbol{k}^{\prime}}$, is given by

$$
\hat{\boldsymbol{x}}_{k}=\overline{\boldsymbol{x}}_{k}+K_{k}\left(\boldsymbol{y}_{k}-D \bar{x}_{k}\right)
$$

(4) The covariance matrix of $\hat{\boldsymbol{x}}_{k}$ is computed by:

$$
\hat{X}_{k}=\left(I-K_{k} D\right) \bar{X}_{k}
$$

(5) The state vector at time $k+1$ is predicted by:

$$
\overline{\boldsymbol{x}}_{k+1}=\boldsymbol{f}\left(\hat{\boldsymbol{x}}_{k^{\prime}}, u_{k}, \overline{\boldsymbol{v}}_{k}\right)
$$

where

$f$ state transition vector function

$u_{k}$ control signal

$\vec{v}_{k}$ measured disturbances

(6) The covariance of $\overline{\boldsymbol{x}}_{k+1}$, is computed by

$$
\bar{X}_{k+1}=\Phi_{k} \hat{X}_{k} \Phi_{k}^{\mathrm{T}}+V
$$

where

$$
\begin{gathered}
\Phi_{k}=\partial f / \partial x_{k} \mid \\
x_{k}=\hat{x}_{k} \\
V \quad \text { covariance matrix of process noise }
\end{gathered}
$$

(7) $k:=k+1$ and return to (2) 